CERN-TH/2001-258

$\mathrm{T} 01 / 103$

hep-th/0109207

\title{
Quintessence Model Building
}

\author{
Ph. Brax円 \\ CERN, Theory Division, CH-1211 Geneva 23, Switzerland. \\ J. Martin \\ Institut d'Astrophysique de Paris, 98 Boulevard Arago, 75014, Paris, \\ France. \\ A. Riazuelo \\ Département de Physique Théorique, Université de Genève, 24 Quai \\ Anselmet 1211 Genève-4, Switzerland.
}

\begin{abstract}
A short review of some of the aspects of quintessence model building is presented. We emphasize the role of tracking models and their possible supersymmetric origin.
\end{abstract}

\section{Introduction}

The cosmological constant problem is one of the thorniest issues of present day theoretical physics [1]. It has recently received a new twist from the analysis of the luminosity distance vs redshift diagram of type Ia supernovae 22 combined with the cosmic background anisotropy results [3, 4, 5, 6]. It appears that our universe seems to be in a late accelerated phase of expansion driven by a vacuum energy density of the order of the critical energy density of the universe. This is strongly suggested by the observation of a negative

\footnotetext{
${ }^{1}$ On leave of absence from Service de Physique Théorique, CEA-Saclay, F-91191, Gif/Yvette cedex, France
} 
acceleration parameter $q_{0}$ and the location of the first Doppler peak, a result compatible with a spatially flat universe in agreement with the inflationary scenario.

From a theoretical point of view this poses two highly different cosmological constant problems. The first one corresponds to the lack of explanation for an almost exact vanishing of the cosmological constant. Indeed modern theories of particle physics predict that radiative corrections and phase transitions such as the one occurring at the weak scale, the scale of the electroweak symmetry breaking, or even worse the Planck scale, contribute to the fourth power to the vacuum energy. Without the need to invoke any putative physics beyond the standard model of particle physics one can see that a term like $M_{W}^{4} \simeq 10^{8} \mathrm{GeV}^{4}$ destroys the delicate balance required by the almost vanishing of the cosmological constant. Within the realm of high energy physics such an acute cancellation between diverse sources of vacuum energy appears as a fine-tuning of the parameters of the theory and requires a dynamical explanation. One of the most commonly advocated mechanisms is the presence of a symmetry which would naturally lead to the vanishing of the cosmological constant [7].

Supersymmetry is such a symmetry [8]. As a matter of fact globally supersymmetric theories have a vanishing vacuum energy [9]. Unfortunately our world does not seem to be described by a globally supersymmetric theory; despite intensive searches, supersymmetric partners of the leptons such as the selectrons have never been observed. This is usually accommodated by requiring that supersymmetry is softly broken therefore losing the exact vanishing of the cosmological constant. Moreover while incorporating gravity in the supersymmetric setting, i.e. supergravity, one finds that supersymmetric vacua do not automatically lead to a vanishing vacuum energy. Supergravity vacua tend to favor a negative cosmological constant and various no-go theorems forbid the existence of de Sitter or accelerating supergravity vacua 11. Similarly string theory 10 in its usual form cannot be formulated in de Sitter or accelerating universes due to the presence of a horizon, and the impossibility of defining a scattering S-matrix [12].

The quintessence proposal does not address the previous hard cosmological constant problem 13, 14. It tackles the issue of obtaining a small and non-vanishing cosmological constant provided that an unknown mechanism guarantees that the hard cosmological constant problem is solved. In practice this amounts to studying field-theoretical models involving one or several fields evolving in time under the combined action of gravity and field interac- 
tions. Quintessence arises when the present value of the potential is compatible with a fraction $\Omega_{Q} \simeq 0.7$ of the critical energy density $\rho_{\mathrm{c}} \simeq 10^{-48} \mathrm{GeV}^{4}$ of the universe. Notice that this involves finding models where the vacuum energy of the universe is some hundred and twenty orders of magnitude smaller than the Planck scale. Of course most models with such a large hierarchy of scales will suffer from some kind of fine-tuning. Fortunately there exists a class of models, the tracking models, where the quintessence field rolls down a potential while converging towards an attractor 15. This tracking mechanism leads to small values of the vacuum energy without invoking a fine-tuning of the initial conditions. Still it requires the choice of a particular scale $M$ for the quintessence sector. Such a scale can be chosen to be large, for instance $10^{10} \mathrm{GeV}$, therefore avoiding the need for an arbitrary small scale in the model. Of course it would be nice if the choice of this quintessence scale could be linked to some more fundamental scales such as the Planck scale or the string scale.

As already mentioned the best possible candidates for solving the hard cosmological constant problem is supergravity, or string theory. Nevertheless it seems that finding accelerating supersymmetric vacua in these theories is an impossible task. Of course this is not the case anymore when supersymmetry is broken. One suggestive possibility is that supersymmetry is broken while preserving a vanishing cosmological constant, this could happen for instance as the result of an accidental cancellation. In that setting one can try to implement the quintessence proposal and look for quintessence models with broken supersymmetry. We will present some examples here[16].

In a first section we will recall the four distinct problems that quintessence models should address. Then we will present examples of simple quintessence models, such as exponential models. Eventually we will introduce tracking models and discuss the issue of supersymmetric tracking models [16, 17, 18].

\section{Quintessential Problems}

In the following we will restrict ourselves to quintessence models with a single quintessence field $Q$. More precisely the dynamics of this scalar field will be governed by the Friedmann and Klein-Gordon equations. The pressure and energy density of a scalar field are defined by

$$
p_{Q}=\frac{\dot{Q}^{2}}{2}-V(Q), \rho_{Q}=\frac{\dot{Q}^{2}}{2}+V(Q),
$$


where $V(Q)$ is the potential energy of the quintessence field. The evolution of the scale factor of the universe $a$ is dictated by the Friedmann equation

$$
H^{2} \equiv\left(\frac{\dot{a}}{a}\right)^{2}=\frac{\kappa}{3} \rho,
$$

where $\rho$ is the total energy density involving cold dark matter, radiation and the quintessence field. Notice that we have assumed an exactly vanishing cosmological constant. The Klein-Gordon equation gives the time evolution of the quintessence field

$$
\ddot{Q}+3 H \dot{Q}=-\frac{\partial V(Q)}{\partial Q}
$$

The phenomenological constraint on $\rho_{Q}$ is that it should correspond to an energy scale of order $5.7 \times 10^{-47} \mathrm{GeV}^{4}$ now.

\subsection{The Coincidence problem}

The cosmological coincidence problem is the "why now" question, i.e. why should we observe a slight dark energy dominance compared to the other forms of matter now? In the context of quintessence this can be reformulated as follows. Why does the evolution of the quintessence field leads to dominance now? In particular one should be wary of a very serious initial conditions problem. Indeed it would be extremely unnatural to assume that coincidence occurs due to a particular choice of the initial conditions for $Q$. For that reason tracking quintessence models are favored. They lead to the independence of coincidence with respect to the initial conditions. This is due to the presence of an attractor.

Another coincidence is that dark energy dominates soon after matterradiation equality. This is often dubbed as the triple coincidence problem. Assuming that cold dark matter is due to a stable particle with interactions governed by a scale $M_{\mathrm{CDM}}$ it has been observed that the energy density at

matter-radiation equality scales as $M_{\mathrm{CDM}}^{8} / m_{\mathrm{Pl}}^{4} 19$. In order to have a natural explanation for the triple cosmic coincidence this requires that

$$
\left.\rho_{Q}\right|_{\text {now }} \simeq \frac{M_{\mathrm{CDM}}^{8}}{m_{\mathrm{Pl}}^{4}},
$$

where $M_{\mathrm{CDM}} \simeq 10^{3} \mathrm{GeV}$. This suggests that a see-saw mechanism is at play. 


\subsection{The Fine-Tuning Problem}

Any choice of potential does not lead to a valid quintessence model. Indeed one should avoid unnatural choices of parameters. For instance take the potential $V(Q)=m^{2} Q^{2} / 2$ where the only free parameter must be chosen to be $m \simeq 10^{-33} \mathrm{eV}$, a scale as difficult to explain as a small vacuum energy. As already mentioned, fom tracking models, the potential depends on a single scale $M$

$$
V(Q)=\frac{M^{4+\alpha}}{Q^{\alpha}}
$$

Depending on $\alpha$, the value of $M$ has to chosen in order to obtain $\Omega_{Q}=0.7$ now. For instance for $\alpha=6$ one finds $M=10^{6} \mathrm{GeV}$. Observe that such a choice of $M$ can be viewed as a fine-tuning, why should physics involve such an energy scale? On the other hand one can argue that this does not involve any fine-tuning as such an energy scale is compatible with high energy

physics. Granted such a value for $M$ we are guaranteed that coincidence occurs now in a way totally independent of the initial conditions.

\subsection{The equation of state}

Another phenomenological constraint on quintessence models concerns the equation of state

$$
\omega_{Q} \equiv \frac{p_{Q}}{\rho_{Q}} .
$$

For a pure cosmological constant $\omega_{\Lambda}=-1$ while recent data suggest $\omega_{Q} \leq$ -0.8 . This is a very stringer restriction on various types of models. Most models will fail to satisfy this criterion.

\subsection{Model Building}

One would like to find natural quintessence models which can be embedded in high energy physics. Eventually one would like to understand quintessence as emerging from string theory. One interesting possibility is to consider the fate of moduli in string theory, i.e. fields like the dilaton. As a rule these fields have no potential perturbatively while a non-vanishing runaway potential is generated non-perturbatively. It is tempting to think of the quintessence field as one of these moduli. In the case of the dilaton the potential is of 
the exponential of exponential type and does not lead to an appropriate potential[20]. More work along these lines will certainly be fruitful.

Another possibility is to try to link the quintessence field to the breaking of supersymmetry. Of course this requires to parameterize the spontaneous breaking of supersymmetry 21]. We will present such an example.

\section{Some Quintessence Models}

In order to illustrate the previous considerations we will consider two typical examples. In particular we will pay attention to the initial conditions problem. This problem is solved provided 22, 23]

$$
\Gamma=\frac{V(Q) V^{\prime \prime}(Q)}{V^{\prime}(Q)^{2}}>1
$$

during the evolution of $Q$. This guarantees the existence of an attractor and the loss of sensitivity to the initial conditions.

\subsection{Pseudo Goldstone Boson Model}

Consider the potential which can arise for very light axions after the breaking of the Peccei-Quinn symmetry[24]

$$
V(Q)=M^{4}\left[\cos \left(\frac{Q}{f}\right)+1\right]
$$

There are two scales in this potential. The scale $f$ is the symmetry breaking scale which is chosen to be $f \geq 10^{16} \mathrm{GeV}$. Starting with $Q \ll f$ we see that $Q$ will roll down towards the minimum of the potential. The scale $M$ must be chosen to be small enough in order to reach $\Omega_{Q}=0.7$ now. For instance one can take $M \simeq 10^{-1} \mathrm{eV}$. Of course this is a fine-tuning which receives no explanation here and jeopardizes the predictability of the model ${ }^{2}$. Moreover the initial conditions must be tuned to reach the right vacuum energy now 25]. This can be seen by noticing that $\Gamma$ becomes much smaller than one when $Q$ is close to the minimum of the potential.

\footnotetext{
${ }^{2}$ See 19 for a model where $M$ is related to the supersymmetry breaking scale in a hidden sector.
} 


\subsection{Exponential Potentials}

These potentials have the nice feature of being naturally generated by compactifying higher dimensional supergravities 26]. The potential

$$
V(Q)=V_{0} e^{-\lambda Q}
$$

depends on one scale $V_{0}$ and a constant $\lambda$. Due to the exponential behavior large scale hierarchies can be easily generated for a high value of $V_{0}$. For a large range of initial conditions this model possesses an attractor which tracks the behavior of the dominant matter or radiation energy density [27]. Unfortunately such an attractor leads to an effective equation of state for the quintessence field which coincides with the one of the tracked matter density. At late times this means $\omega_{Q}=0$ and therefore no acceleration of the universe. This can be remedied by choosing the initial conditions outside of the basin of attraction of the attractor. This has the unwanted feature of leading to a dependence on the initial conditions. The initial conditions problem can be remedied by double exponential potentials.

\section{Tracking Quintessence Models}

These models preserve the nice tracking property of exponential potentials while leading to a negative equation of state and late acceleration. As $\Gamma>$ 1 the late evolution of the quintessence field is independent of the initial conditions. The quintessence field reaches an attractor when either radiation or matter dominates and leaves the attractor in the recent past to start dominating over the CDM matter density. The only parameter $M$ has to be tuned in order to reach the appropriate value of $\Omega_{Q}$ now.

These models can be obtained in a supersymmetric context by considering a super-Yang-Mills theory with gauge group $S U\left(N_{c}\right)$ and $N_{F}<N_{c}$ quark flavors 20. At energies below the gluino condensation scale $M$ a nonperturbative superpotential for the meson fields is generated. It leads to a inverse power law potential. In this model a large condensation scale like $10^{6} \mathrm{GeV}$ for $\alpha=6$ is not unnatural.

These models suffer from two main problems. On the one hand the first problem springs from the observation that they lead to an equation of state $\omega_{Q}$ which is always larger that -0.7 , i.e. falling outside of the experimental ball-park. 
On the other hand the supersymmetric tracking model is not consistent unless one takes supergravity into account 16. Indeed this can be easily seen by noticing that on the attractor, and therefore just before coincidence, one finds

$$
V^{\prime \prime}=\frac{9}{2} \frac{\alpha+1}{\alpha}\left(1-\omega_{Q}^{2}\right) H^{2},
$$

where $V^{\prime \prime}$ is the second derivative of the potential. Using Friedmann's equation, this leads to

$$
\left.Q\right|_{\text {now }} \simeq m_{\mathrm{Pl}} .
$$

A globally supersymmetric theory receives supergravity corrections in the form of $Q / m_{\mathrm{Pl}}$ which cannot be neglected in the late stage of the evolution of the universe. In particular a naive calculation of the supergravity potential for the gaugino condensation model $l^{3}$ leads to a negative energy density in the recent past.

We therefore conclude that the description of quintessence by tracking fields requires an embedding of the model in supergravity where terms in $Q / m_{\mathrm{Pl}}$ are properly taken into account.

\section{$5 \quad$ Supergravity Tracking Models}

\subsection{The Supergravity Tracking Potential}

We consider the possibility that tracking models arise from supergravity in four dimensions. Eventually these models must be considered as an effective field theory description of a more fundamental theory. We will therefore only concentrate on describing mechanisms leading to tracking potentials in four dimensional supergravity leaving an explanation for the origin of these models to more refined studies. As a rule the potential in supergravity depends on a single function $G=\kappa K+\ln \left(\kappa^{3}|W|^{2}\right)$ where $K$ is the Kähler potential governing the kinetic terms $K_{i \bar{j}} \partial_{\mu} \phi^{i} \partial^{\mu} \bar{\phi}^{j}$ and $W$ is the superpotential. The scalar potential reads

$$
V(Q)=\kappa^{-1} e^{G}\left(G^{i} G_{i}-3\right)+V_{\mathrm{D}}
$$

where $V_{\mathrm{D}}$ is positive definite and involves the gauge sector of theory. In the following we consider flat $D$ directions where $V_{\mathrm{D}}$ vanishes altogether. Globally supersymmetric theories can be obtained by letting $\kappa \rightarrow 0$. In that case

\footnotetext{
${ }^{3}$ We assume a flat Kähler potential.
} 
the potential becomes positive definite. Consider the case of quintessence with a low value of the initial condition for $Q$. In that case supergravity corrections are small and one can trust the globally supersymmetric approximation. Assume that the models leads to an inverse power law model for low values of $Q$. As $Q$ evolves towards the Planck scale, the main supergravity correction will come from the prefactor $e^{\kappa K}$ which leads to the supergravity corrected potential 16

$$
V(Q)=e^{\frac{\kappa}{2} Q^{2}} \frac{M^{4+\alpha}}{Q^{\alpha}}
$$

Notice that the exponential correction does not play a role for most of the evolution of $Q$. It only becomes relevant when the quintessence field dominates leading to a rapid increase of the potential energy. This has the nice phenomenological feature of leading to an equation of state $\omega_{Q} \simeq-0.82$ almost independently of $\alpha$.

\section{$5.2 \quad$ A Supergravity Model}

The construction of supergravity quintessence models necessitates the existence of three independent sectorst the observable sector where the standard model fields live, a supergravity breaking sector where the superpartners acquire their masses and a quintessence sector where supersymmetry is also broken[17]. The three independent sectors can be coupled by gravity for instance. The quintessence sector must be decoupled from the observable sector as the effective mass of the quintessence field now, see Eq. (10), is of the order of the Hubble parameter $H_{0} \simeq 10^{-43} \mathrm{GeV}$. Such a small mass would lead to the existence of a long range fifth force contradicting experimental facts 28. The quintessence sector and the supersymmetry breaking sectors must be decoupled to prevent the existence of time dependent sparticle masses [17]. If time-dependent sparticle masses are allowed the large contribution of the quintessence field $Q$ to the supersymmetry breaking $F$-terms might lead to very large values of the sparticle masses, preventing any possible detection. We discard this possibility here.

Let us now consider a typical string inspired scenario involving type I models 29. Such models comprise branes where interactions are set by the string scale $m_{\mathrm{S}}$ and suppressed gravity effects 30. Moreover they contain many anomalous $U(1)$ gauge groups with associated Fayet-Iliopoulos

\footnotetext{
${ }^{4}$ We acknowledge an interesting discussion with P. Binétruy on that issue.
} 
terms [31] of the order of the string scale.

At low energy the corresponding (broken) supergravity models will possess two characteristic scales, the string scale $m_{\mathrm{S}}$ that we suppose to be much smaller than the Planck scale. We can construct a simple supergravity quintessence model by using three fields, the quintessence field $Q$ and two fields $X$ and $Y$ of charges 1 and -2 under an anomalous $U(1)$ gauge group. We assume that the leading interaction between $X$ and $Y$ is given by the superpotential $W=X^{2} Y$. The model possesses a D-flat direction where $Y=0$ and $X=m_{\mathrm{S}}$ due to the presence of a non-vanishing Fayet-Iliopoulos term. Moreover we assume that the Kähler potential takes the form

$$
K=Q \bar{Q}+Y \bar{Y} f\left(Q, \bar{Q}, m_{\mathrm{S}}, m_{\mathrm{Pl}}\right)+X \bar{X} .
$$

Notice that we have a non-trivial coupling between $Y$ and $Q$ in the Kähler potential. Due to the large hierarchy between the string and Planck scales we take $f\left(Q, \bar{Q}, m_{\mathrm{S}}, m_{\mathrm{Pl}}\right) \equiv f\left(Q \bar{Q} / m_{\mathrm{S}}^{2}\right)$ to be a positive increasing function normalized by $f(0)=1$. For $Q \ll m_{\mathrm{S}}$ we can expand $f$ in power series. For larger values non-perturbative information might be necessary. We assume that $f$ can always be defined by a polynomial of degree $p$. It is now an easy exercise to compute the scalar potential

$$
V(Q)=e^{\kappa Q^{2} / 2} m_{\mathrm{S}}^{4} f^{-1}\left(\frac{Q \bar{Q}}{m_{\mathrm{S}}^{2}}\right) .
$$

For small values of $Q \ll m_{\mathrm{S}}$ the potential is almost constant $V(Q) \simeq m_{\mathrm{S}}^{4}$. For larger values of $Q$ the potential decreases before reaching large values of $Q$ where the exponential supergravity correction becomes relevant. Notice that the potential energy is always smaller than $m_{\mathrm{S}}^{4}$ as necessary for the low energy effective action approach to be valid.

Assuming that $f$ is polynomial at large $Q$ leads to

$$
V(Q) \simeq e^{\frac{\kappa}{2} Q^{2}} \frac{m_{\mathrm{S}}^{4+2 p}}{Q^{2 p}},
$$

where we have chosen $Q=\bar{Q}$ [. Notice that this is the supergravity tracking potential with $\alpha=2 p$. So by allowing $Q$ to roll down the potential (15)

\footnotetext{
${ }^{5}$ These are moduli associated to the blowing up modes of the orbifold compactification. We assume that they are somehow stabilized at a value of the order of the string scale.

${ }^{6}$ See the related talk by R. Caldwell for a discussion of spinessence.
} 
we find that the quintessence potential behaves effectively like a tracking potential before feeling the effect of the supergravity corrections. Moreover choosing the string scale to be of order of the TeV scale leads to $\alpha=4$. Putting $Q \simeq m_{\mathrm{Pl}}$ now we find that

$$
\left.\rho_{Q}\right|_{\text {now }}=\frac{m_{\mathrm{S}}^{8}}{m_{\mathrm{Pl}}^{4}},
$$

as suggested in order to solve the triple coincidence problem with $M_{\mathrm{CDM}}=$ $m_{\mathrm{S}} \simeq 10^{3} \mathrm{GeV}$.

In conclusion we have described a simple string inspired supergravity model leading to a tracking model with interesting phenomenological fea-

tures. Of course much work remains to be done to make this picture more explicit.

\section{Conclusion}

We have presented some of the aspects of quintessence model building putting the emphasis on the necessity of constructing models embedded in high energy theories such as supergravity. The subject of quintessence model building is still in its infancy and is likely to receive new impetus from string theory and its various avatars.

\section{Acknowledgments}

One of us (P.B.) thanks the organizers of the conference for kindly allowing him to present this work.

\section{References}

[1] S. Weinberg, Rev. Mod. Phys. 61, 1 (1989).

[2] S. Perlmutter et al., Nature 391, 51 (1998); S. Perlmutter et al., Astrophys. J. 517, 565 (1999); P.M. Garnavich et al., Astrophys. J. 493, L53 (1998); A.G. Riess et al., Astron. J. 116, 1009 (1998).

[3] P. de Bernardis et al., Nature 404, 955 (2000). 
[4] A.E. Lange et al., Phys. Rev. D 63, 042001 (2001).

[5] S. Hanany et al., Astrophys. J. 545, L5 (2000).

[6] A. Balbi et al., Astrophys. J. 545, L1 (2000).

[7] E. Witten, Lecture given at DM2000, preprint hep-ph/0002297.

[8] Supersymmetry, edited by S. Ferrara, (Elsevier, Amsterdam and World Scientific, Singapore, 1987).

[9] E. Witten, Nucl. Phys. B185, 513 (1981).

[10] J. Polchinski, String Theory, Cambridge University Press (Cambridge, England, 1998).

[11] J. Maldacena and C. Nunez, Int. J. Mod. Phys. A16, 822 (2001).

[12] S. Hellerman, N. Kaloper and L. Susskind, JHEP 0106, 003 (2001).

[13] L. Wang, R.R. Caldwell, J.P. Ostriker and P.J. Steinhardt, Astrophys. J. 530, 17 (2000).

[14] C. Wetterich, Astron. Astrophys. 301, 321 (1995).

[15] B. Ratra and P.J.E. Peebles, Phys. Rev. D 37, 3406 (1988).

[16] P. Brax and J. Martin, Phys. Lett. 468B, 40 (1999).

[17] P. Brax, J. Martin and A. Riazuelo, hep-ph/ 0104240, to appear in Phys. Rev. D.

[18] E.J. Copeland, N.J. Nunes and F. Rosati, Phys. Rev. D 62, 123503 (2000).

[19] N. Arkani-Hamed, L. Hall, C. Kolda and H. Murayama, Phys. Rev. Lett. 85, 4434 (2000).

[20] P. Binétruy, Phys. Rev. D 60, 063502 (1999).

[21] A. Brignole, L.E. Ibanez and C. Munoz, Nucl. Phys. B 422, 125 (1994).

[22] P.J. Steinhardt, L. Wang and I. Zlatev, Phys. Rev. D 59, 123504 (1999). 
[23] I. Zlatev, L. Wang and P.J. Steinhardt, Phys. Rev. Lett. 82, 896 (1999).

[24] J. Frieman et al., Phys. Rev. Lett. 752077 (1995).

[25] A. Albrecht and J. Weller, astro-ph/0106079.

[26] P.M. Cowdall, H. Lu, C.N. Pope, K. Stelle and P.K. Townsend, Nucl. Phys. B 48649 (1997).

[27] P.G. Ferreira and M. Joyce, Phys. Rev. D 58, 023503 (1998).

[28] A. Masiero, M. Pietroni and F. Rosati, Phys. Rev. D 61, 023504 (2000).

[29] I. Antoniadis, N. Arkani-Hamed, S. Dimopoulos and G. Dvali, Phys. Lett. 436B, 257 (1998).

[30] L.E. Ibanez, C. Munoz and S. Rigolin, Nucl. Phys. B 553, 43 (1999).

[31] M. Dine, N. Seiberg and E. Witten, Nucl. Phys. B 289, 585 (1986). 